\title{
Ultrasound in critical care
}

\author{
Paweł Andruszkiewicz ${ }^{1,3}$ Dorota Sobczyk ${ }^{2,3}$ \\ ${ }^{1} 2^{\text {nd }}$ Department of Anaesthesiology and Intensive Therapy, Medical University of Warsaw, Poland \\ ${ }^{2}$ John Paul II Specialist Hospital in Cracow, Poland \\ ${ }^{3}$ Working Group of Ultrasonography and Echocardiography, Polish Society of Anaesthesiology \\ and Intensive Therapy, Criticalusg
}

\begin{abstract}
Ultrasound has been revolutionizing our specialty. Introduction of new, portable machines and goal directed protocols has led many anaesthetists to use this diagnostic tool in their daily practice.

Immediate, bedside ultrasound diagnosis of many life-threatening emergencies (pneumothorax, cardiac tamponade, or internal haemorrhage) enables not only institution of proper treatment, but also monitoring of its effectiveness. Ultrasound guided invasive procedures (such as vascular cannulations, toraco- and pericardiocentesis) supersede the old anatomical landmarks-based techniques due to increased safety margin.

In order to perform credible ultrasound examination, proper level of competence is required. In this review article authors present the variety of critical ultrasound aplications.
\end{abstract}

Key words: intensive therapy, diagnostics; intensive therapy, ultrasound; intensive therapy, echocardiography

Anaesthesiology Intensive Therapy 2013, vol. 45, no 3, 177-181

Ultrasound-based diagnostic procedures have been used in departments of anaesthesiology and intensive therapy for many years. They were formerly the domain of radiologists (US) and cardiologists (echocardiography). In recent years, ultrasound technology has been increasingly employed by physicians of other specialties, including anaesthesiologists, which is undoubtedly associated with its huge and comprehensive potential as well as the latest technological advances. Portable, miniaturised ultrasound devices currently available allow immediate, bedside diagnoses of many life-threatening diseases without transporting patients to diagnostic units.

The ultrasound machine characteristic particularly useful for anaesthesiologists is the time required to achieve its readiness for use, which can be of utmost importance when used for resuscitation. Some ultrasound devices are ready for use within only several seconds. Modern devices are equipped with the user-friendly software and technology facilitating imaging by less experienced sonographists. Recognising the specific needs of our professional group, some producers designed the devices with built-in software for self-education. During the procedure, physicians can revise their knowledge of topographic anatomy of a given body region on the screen and see the animation facilitating ultrasound procedures or ultrasound-guided regional blocks.

Modern ultrasound devices are additionally equipped with the systems allowing data archiving in the form of pictures or short video clips. The recorded data can be replayed and transferred to memory cards. Moreover, the systems enabling real-time transmission of ultrasound images have recently been designed. Such a system was successfully employed to transmit to Earth the research carried out by an astronaut at the space station or events in the Himalayan expedition camp [1]. Less experienced sonographists find telemedicine very handy to consult more experienced specialists, if need be.

In the ICU filled with medical equipment, small sizes of devices are desirable enabling examinations in very small spaces available. Moreover, such features as impact resistance (falls from height) or easy cleansing (e.g. flat keyboards), which reduces the risk of infection spreading, are useful [2].

In comparison with other widely used diagnostic modalities, such as radiographic examinations or computed 
tomography, ultrasound imaging does not require the use of ionizing radiation, which can be relevant for patients requiring long-term therapy and multiple exposures.

\section{ECHOCARDIOGRAPHY}

Echocardiography has traditionally been the domain of cardiologists. One of the reasons is a widespread belief that the technique is extremely complicated. The situation changed diametrically once small, portable US devices were introduced and simplified protocols of transthoracic echocardiographic examinations were implemented. The protocols such as focus assessed transthoracic echocardiography (FATE) or focused echocardiographic evaluation in life support (FEEL) [3-6] were dedicated for the users who were not continuously dealing with echocardiography or cardiology. The protocols mentioned enabled to limit to a minimum the number of projections, to use only basic techniques (in practice, 2D) and to focus on a particular spectrum of lesions.

It has soon been realised that echocardiography is the tool almost indispensible for intensive care units or emergency departments. Transthoracic echocardiography is a non-invasive, bedside and burdenless examination. The majority of cardiologic life- threatening conditions, e.g. pericardial tamponade, hypovolaemia, aortic dissection, myocardial infarction or acute right ventricular failure, can be detected (or excluded) using focused echocardiography. It can also be used to monitor the therapy administered and during interventional procedures (e.g. pericardiocentesis) [4, 5]. Moreover, the use of basic echocardiographic diagnostic procedures during cardiopulmonary resuscitation has been increasingly recommended. This is reflected in the guidelines of the European Resuscitation Council of 2010 [7], which allow using them during the 10-second interval to assess pulse when pulseless electric activity is suspected. The FEEL protocol enables to detect some potentially reversible causes of cardiac arrest [6].

Furthermore, the use of transoesophageal echocardiography (TEE) by anaesthesiologists is being widely discussed. However, it should be remembered that TEE is a half-invasive method and should be used only for certain clinical indications. In the ICU setting, this examination is usually confined to cases in which the transthoracic echocardiograms are non-diagnostic or when transthoracic examinations are technically unfeasible (e.g. due to extensive thoracic injuries). The range of applications of TEE is distinctly wider in the operative suite setting. TEE is routinely used in cardiac surgery during valve corrective repairs and valve replacement procedures of low invasiveness or percutaneous ones [8]. In some centres (e.g. Toronto General Hospital), intraoperative transoesophageal echocardiography is routinely performed for monitoring haemodynamic status during cardiac surgeries. Moreover, there are seve- ral indications for planned use of TEE during non-cardiac surgeries, such as neurosurgical procedures (detection of venous air embolism), lung and liver transplantations and procedures in patients at high cardiovascular risk (e.g. with markedly reduced left ventricular ejection fraction). The key indication for emergency TEE in the operative suite is still sudden onset hypotonia (particularly in patients with heart conditions cardiac problems) [4].

Echocardiography is undoubtedly one of the most difficult areas of ultrasonography. The interpretation of results requires much experience, in-depth knowledge of anatomy, physiology and cardiology as well as continuous trainings [9].

\section{LUNG ULTRASOUND}

Until recently, radiologists have believed that ultrasonography is useless for the diagnosis of lung diseases, basing on the assumption that sound waves are completely dispersed in the aerated lungs; thus, imaging relying on interpretation of characteristics of a sound wave reflected from objects is not possible [10]. The long-term observations of Daniel Lichtenstein, a pioneer of ultrasound-based lung diagnostic procedures, compelled them to revise their opinion. According to Lichtenstein, in many pulmonary and pleural diseases in which the air-to-fluid compartment ratio is altered, ultrasound scans contain characteristic artefacts whose qualitative and quantitative interpretation enables clinical diagnosis [11]. Ultrasound as a bedside, portable diagnostic tool is perfectly suited for differential diagnosis of ailments that lead to the development of acute respiratory failure [11-17].

An excellent example of a simplified goal directed at identification of the cause of respiratory failure is the bedside lung ultrasonography in emergency (BLUE) protocol proposed by Lichtenstein [11]. During the several-minute examination, the placement of the transducer probe over several thoracic areas enables to diagnose or exclude pneumothorax, pleural effusion, pulmonary oedema or pneumonia. Proper diagnosis and immediate institution of causal therapy may be essential for the fate of patients with severe dyspnoea. History taking and physical examinations do not always allow establishing accurate diagnoses. Radiological examinations and computed tomography require time or transportation of patients to diagnostic units, which can be infeasible due to their conditions.

Numerous studies [11, 15-19] have demonstrated that lung ultrasound is more sensitive and specific than chest radiography and only slightly inferior to computed tomography. A tremendous asset of ultrasonography is its possible repetition during the therapeutic process to verify the efficacy of treatment and to monitor the subsidence of symptoms. An example of this kind of ultrasound use is the assessment of lung expansion after pleural drainage or consolidation size decrease following PEEP [19]. 
Nonetheless, it should be highlighted that lung ultrasound has its limitations. The pathological areas can be visualised only when the sound-transmitting medium is present between the lesion and the transducer probe. Air is an impassable barrier for sound waves. Thus, even a large lung abscess will be „invisible" when surrounded by the aerated pulmonary parenchyma. For similar reasons, subcutaneous emphysema is a diagnostic impediment [20].

\section{OCULAR ASSESSMENT}

Ultrasound examinations can be extremely useful diagnostic tools for patients with head injuries, particularly when the eyeball damage is suspected. The pathologies such as a foreign body in the vitreous humour, traumatic lens displacement or retinal detachment can be easily visualised within the several-minute examination [21]. Moreover, with some experience gained, the pupillary light response can be assessed. Ultrasonography is particularly helpful when the ophthalmic examination is hindered or infeasible due to eyelid oedema.

The assessment of the optic nerve sheath diameter can enable the bedside, non-invasive detection of increased intracranial pressure (ICP) [21-24]. The optic nerve sheath is a "projection" of the intracranial subarachnoid space; therefore, increased ICP can lead to its widening [21, 25, 26]. The examination in question is performed using a linear probe evaluating the sheath width at a defined retrobulbar point $(0.3 \mathrm{~cm})$. Noteworthy, ultrasound assessment does not replace invasive measurements of ICP, which remain the gold standard [27].

\section{ASSESSMENT OF PARANASAL SINUSES}

Ultrasonography can also be used to confirm paranasal sinusitis [28]. It is estimated that fever is likely to be caused by sinusitis in one in three patients undergoing artificial ventilation. Physiologically, sinuses are filled with air hence are invisible to ultrasound. When inflamed, the fluid occurs in them forming the medium for transmission of sound waves and imaging $[29,30]$. The limitation of this method is the lack of the "bone window" indispensible for sound wave transmission. However, in many individuals, the thickness of the anterior maxillary sinus wall is not an impediment for imaging. Sinus ultrasound is characterised by relatively high sensitivity and specificity [31].

\section{FOCUSED ASSESSMENT WITH SONOGRAPHY FOR TRAUMA (FAST)}

FAST is a simplified protocol of ultrasound examination used in patients with blunt abdominal injuries, which is directed at identification of free fluid in body cavities (the peritoneum, pericardium, pleura). It is not aimed at detecting the cause of haemorrhage or evaluating the damage to a particular parenchymatous organ. In the context of clinical trauma, the detection of free fluid indicates the presence of blood. [32, 33].

FAST involves the evaluation of four projections: the Morrison's pouch, perisplenic space, substernal region (for the presence of fluid in the pericardial sac) and pouch of Douglas in females or rectovesical pouch in male patients (fluid in the pelvis). Its widened version, extended FAST (e-FAST), is applied for assessment of pleural cavities to exclude pneumothorax or confirm the presence of fluid (blood).

FAST is particularly relevant for haemodynamically unstable patients when time-consuming CT-based diagnosis is likely to be associated with exposing them to risk. Under such circumstances, the confirmation of free fluid in the abdominal cavity should be the sufficient indication for emergency surgery. In recent years, FAST has gained enormous popularity. Thanks to its simplicity, the protocol is also successfully applied for pre-hospital diagnoses (e.g. at the battlefields by military paramedics). However, the method has its caveats; one of them is its sensitivity. The diagnostic difficulties are also faced in cases of organised haematomas whose echogenicity differs from the picture of fresh blood and can resemble that of a parenchymatous organ.

The FAST protocol is a screening test performed in emergency cases, which should be promptly complemented by meticulous ultrasound scanning carried out by an experienced sonographer.

\section{VASCULAR CANNULATION}

In recent years, many opinion-forming institutions have published the recommendations advising the use of ultrasound during central venous cannulation [34-36]. Compared to traditional methods based on the identification of anatomical landmarks, ultrasonography has been demonstrated to be associated with lower incidence rates of complications (e.g. unintentional artery puncture) and fewer attempts required for successful puncture. Moreover, the method enables to shorten the time of effective cannulation. UItrasound-guided cannulation of the internal jugular vein is particularly useful in patients with severe coagulation disorders, obesity or when punctures are performed in the atypical, forced position of the patient.

Prior to cannulation, interrelations between vessels and adjacent anatomic structures should be preliminarily assessed. It is essential to confirm the patency of the punctured vein. The American Society of Echocardiography (ASE) recommends real-time US-guided cannulation when the user carries out the procedure under visual control keeping the transducer probe in the other hand [34].

Furthermore, ultrasound can be extremely beneficial to determine the cannula position and to confirm or exclude iatrogenic complications [37]. By manipulating the transdu- 
cer probe, we can visualize the cannula in the vascular lumen and the direction of its translocation to the brachiocephalic vein. If possible, it should be checked whether its tip has not been inserted into the right atrium.

US is a more sensitive and specific tool for detection of pneumothorax than chest X-ray [28, 37]. Ultrasound assessment directed at exclusion of this complication should be performed immediately after cannulation.

\section{OTHER APPLICATIONS OF ULTRASOUND IN INTENSIVE CARE}

The present review discusses only selected, currently most popular applications of ultrasound in intensive care. Anaesthesiologists have been increasingly using this technology as the "stethoscope of the $21^{\text {st }}$ century" enabling vertical, comprehensive, "head-to-toe" assessment of the patient.

\section{US EDUCATION}

Ultrasonography is a unique tool for holistic approach to diagnosis and treatment of patients. However, its proper use requires appropriate performance skills and, more importantly, abilities of proper interpretation of scans. It is especially relevant to life-threatening cases when examinations are not performed by imaging specialists and proper diagnosis is likely to decisively affect the management and fate of patients. Therefore, it is essential for physicians to reach a suitable level of competence in performing ultrasound examinations.

Some countries have organised "critical ultrasound" training systems comprising three stages: courses for gaining skills and mentoring at work as well as theoretical and practical exams [5, 38, 39]. A good example of functioning of such a system is TEE certification organised by the European Society of Cardiology.

A serious limitation of practical training for practicing anaesthesiologists can be restricted access to examinations as for the number of procedures performed and important pathologies encountered. E-learning can be of crucial importance in this respect. The Working Group on Echocardiography and Ultrasonography of the Polish Society of Anaesthesiology and Intensive Therapy have created the Polish-language website www.criticalusg.pl, which continuously provides educational materials and films with relevant pathologies in intensive therapy. Moreover, an excellent educational tool is medical simulation. Thanks to the introduction of technically advanced phantoms-simulators, the ultrasound performance (including transoesophageal echo) and interpretation of scans can be learnt.

Recognising the potential of ultrasound as the "stethoscope of the $21^{\text {st }}$ century", some medical universities in the United States have recently included practical ultrasound classes in the medical curriculum (started during the first year of studies) [40]. To promote the use of this technology, 2013 has been declared the Year of Ultrasound [41].

\section{SUMMARY}

Ultrasonography provides thorough, bedside diagnoses of life-threatening conditions enabling the institution of immediate causal treatment that can change the patient's fate. The examinations repeated at short time intervals facilitate the evaluation of therapy efficacy and monitoring of impaired vital functions. USG can be also successfully used for invasive procedures associated with high risk of iatrogenic complications. The technological revolution and simplified diagnostic protocols resulted in increasingly widespread ultrasound use by ICU physicians.

Ultrasound use in daily ICU practice requires an appropriate level of competence. It seems that the Polish Society of Anaesthesiology and Intensive Therapy should play a pivotal role in promoting ultrasound applications and developing the system of accredited training courses.

\section{References:}

1. http://science.nasa.gov/science-news/science-at-nasa/2005/16feb_ultrasound/.

2. Lichtenstein $D$ : The ultrasound equipment. In: Whole body sonography in the critically ill. Springer Verlag, Berlin Heidelberg 2010: 11-18.

3. Labovitz AJ, Noble VE, Biering $M$, et al.: Focused cardiac ultrasound in the emergent setting: a consensus statement of the American Society of Echocardiography and American College of Emergency Physicians. J Am Soc Echocard 2010; 23: 1225-1230.

4. On Behalf of a Collaborative Working Group of the British Society of Echocardiography: A position Statement: Echocardiography in the critically ill. JICS 2008; 9: 197-198.

5. Price S, Via G, Sloth E, et al.: Winfocus Echo-ICU Group: Echocardiography practice, training and accreditation in the intensive care: dokument for the World Interactive Network Focused on Critical Ultrasound (WINFOCUS). Cardiovasc Ultrasound 2008; 6: 49-84.

6. Price $S$, Uddin $S$, Quinn $T$ : Echocardiography in cardiac arrest. Curr Opin Crit Care 2010; 16: 211-251.

7. Zaawansowane zabiegi resuscytacyjne u osób dorosłych. In:Wytyczne resuscytacji 2010. Polska Rada Resuscytacji, Kraków 2010: 121-122.

8. Otto CM: Intraoperative TEE, In:Textbook of clinical echocardiography $4^{\text {th }}$ ed. Sounders Elsevier, Philadelphia 2009: 448-468.

9. Elbarbary M, Melniker L, Volpicelli G, et al.: Development of evidence-based clinical recommendations and consensus statements In critical ultrasound fi eld: why and how? Crit Ultrasound J 2010; 2: 93-95.

10. Harrison's principles of internal medicine $17^{\text {th }}$ ed. McGraw-Hill; New York 2008

11. Lichtenstein D: Introduction to ultrasound. In: Whole body sonography in the critically ill. Springer Verlag. Berlin Heidelberg 2010: 117-128.

12. Lichtenstein D, Mezere $G A$ : Relevance of lung ultrasound in the diagnosis of acute respiratory failure. The BLUE protocol. Chest 2008; 134: 117-125.

13. Lichtenstein $D$ : Should lung ultrasonography be more widely used In the assessment of acute respiratory disease? Expert Rev Resp Med. 2010; 4: 533-538.

14. Roch A, Bojan M, Michelet $P$, et al:: Usufulness of ultrasonography In prediction of pleural eff usions $>500 \mathrm{ml}$ in patients receiving mechanical ventilation. Chest 2005; 127: 224-232.

15. Soldati G, Testa A, Sher S, et al:: Occult traumatic pneumothorax: diagnostics accuracy of lung ultrasonography in the emergency department. Chest 2008; 133: 204-211.

16. Agricola E; Bove T, Oppizzi M, et al.: "Ultrasound comet-tail images": a marker of pulmonary edema: a comparative study with wedle pressure and extravascular lung water. Chest 2005; 127: 1690-1695.

17. Reissig A, Copetti R, Kroegel C: Current role of emergency ultrasound of the chest. Crit Care Med 2011; 39: 839-845. 
18. Volpicelli G, Elbarbary M, Blaivas $M$, et al.: International evidence-based recommendations for point-of-care lung ultrasound. Intensive Care Med 2012; 38: 577-591.

19. Gardelli G, Feletti F, Gamberini E, et al.: Using sonography to assess lung recruitment in patients with acute respiratory distress syndrome. Emerg Radiol 2009; 16: 219-221.

20. Lichtenstein D: Pneumothorax, In: Whole body sonography in the critically ill. Springer Verlag. Berlin Heidelberg 2010: 163-179.

21. Noble $V$ Nelson B. Ocular ultrasound, In: Manual of emergency and critical care ultrasound, 2nd ed. Cambridge Univ Press 2011: 203-211.

22. Blavias $M$, Theodoro $D$, Sierzenski P: A study of bedside ocular ultrasonography in the emergency department. Acad Emerg Med 2002; 9: 971-979.

23. Blaivas $M$, Theodoro $D$, Sierzenski P: Elevated Intracranial Pressure detected by bedside emergency ultrasonography of the optic nerve sheath. Acad Emerg Med 2003; 10: 376-381.

24. Harbison H, Shah S, Marill K, Noble V: Correlation of optic nerve heath diameter with direct measurement of intracranial pressure. Acad Emerg Med 2008; 15: 201-204.

25. Hansen $\mathrm{HC}$, Helmke $\mathrm{K}$ : Validation of the optic nerve sheath response to changing cerebrospinal fl uid pressure: ultrasound fi ndings during intrathecal infusion tests. J Neurosurg 1997; 87: 34-40.

26. Watanabe A, Kinouchi $H$, Horikoshi T, et al.: Eff ect of intracranial pressure on diameter of the optic nerve sheath. J Neurosurg 2008; 109 : 255-258.

27. Lavinio A, Menon $D$ : Intracranial pressure: why we monitor it, how to monitor it, what to do with the number and what's the future? Curr Opin Anaesthesiol 2011; 24: 117-123.

28. Lichtenstein D: Dead and neck, In: Pneumothorax in: whole body sonography in the critically ill. Springer Verlag. Berlin Heidelberg 2010: 243-252.

29. Puhakka T, Heikkinen T, Makela M, et al.: Validity of ultrasonography In diagnosis of acute maxillary sinusitis. Arch Otolaryngol Head Neck Surg 2000; 126: 1482-1486.

30. Zagolski O, Stręk P: Badanie ultrasonografi czne nosa i zatok przynosowych. Pol Merk Lek 2007; 22: 127-132.
31. Hilbert G, Vargas F, Valentino $R$, et al: Comparison of B-mode ultrasound and computed tomography in the diagnosis of maxillary sinusitis In mechanically ventilated patients. Crit Care Med 2001; 29: 501-506.

32. Noble V, Nelson B: Focused assessment with sonography in trauma (FAST), In: Manual of emergency and critical care ultrasound, $2^{\text {nd }}$ ed. Cambridge Univ Press 2011: 27-60.

33. Killu K, Dulchavsky S, Coba V (ed.): The ICU ultrasound pocket book. Detroit 2010.

34. Troianos C, Hartman G, Glas K, et al.: Guidelines for Performing Ultrasound Guided Vascular Cannulation: Recommendations of the American Society of Echocardiography and the Society of Cardiovascular Anesthesiologists. J Am Soc Echocardiogr 2011; 24: 1291-1318.

35. National Institute for Health and Clinical Excellence. NICE Technology Appraisal No 49: guidance on the use of ultrasound locating devices for placing central venous catheters. http://www.nice.org.uk/nicemedia/live/11474/32461/32461.pdf.

36. Augoustides J, Cheung A: Ultrasound should be the standard of care for central catheter Insertion. J Cardiothorac Vasc Anesth 2009; 23: 720-724.

37. VezzaniA Brusasco C Palermo S, et al: Ultrasound localization of central vein catheter and detection of postprocedural pneumothorax: an alternative to chest radiography. Crit Care Med 2010; 38: 533-538.

38. Marik P, Mayo P: Certifi cation and training in critical care ultrasound. Intensive Care Med 2008; 34: 215-217.

39. Tsui B, Yun V: Ultrasound beyond regional anesthesia: formal training? Can J Anaesth 2011; 58: 499-503.

40. Hoppmann $R$, Rao V, Poston $M$, et al: An integrated ultrasound curriculum (iUSC) for medical students 4-year experience. Crit Ultrasound J 2011;3:1-12.

41. http://www.ultrasound2013.org.

\section{Corresponding author:}

Paweł Andruszkiewicz, MD, PhD

ul. Dźwiękowa 13, 02-857 Warszawa, Poland

tel.: 602100798

e-mail:pawel_andruszkiewicz@cyberia.pl

Received: 17.03.2013

Accepted: 21.06.2013 